\title{
ERRATUM
}

Ling-Ping Lai $\cdot$ Yi-Ning Su $\cdot$ Fon-Jou Hsieh

Fu-Tien Chiang $\cdot$ Jyh-Ming Juang $\cdot$ Yen-Bin Liu

Yi-Lwun Ho · Wen-Jone Chen · San-Jou Yeh

Chun-Chieh Wang · Yu-Lin Ko · Tsu-Juey Wu

Kwo-Chang Ueng $\cdot$ Meng-Huan Lei $\cdot$ Hsuan-Ming Tsao

Shih-Ann Chen · Tin-Kwang Lin · Mei-Hwan Wu

Huey-Ming Lo $\cdot$ Shoei K. Stephen Huang · Jiunn-Lee Lin

\section{Denaturing high-performance liquid chromatography screening of the long QT syndrome-related cardiac sodium and potassium channel genes and identification of novel mutations and single nucleotide polymorphisms}

Published online: 28 January 2006

(C) The Japan Society of Human Genetics and Springer-Verlag 2006

\section{J Hum Genet (2005) 50:490-496}

The name Fon-Jou Hsieh was inadvertently omitted from the list of authors. The name should be added as the third author of the article.

The online version of the original article can be found at http:// dx.doi.org/10.1007/s10038-005-0283-3

\section{L.-P. Lai}

Institute of Pharmacology, National Taiwan University Hospital, Taipei, Taiwan

Y.-N. Su

Department of Medical Genetics, National Taiwan University Hospital, Taipei, Taiwan

F.-J. Hsieh

Department of Obstetrics and Gynecology,

National Taiwan University Hospital,

Taipei, Taiwan

F.-T. Chiang $\cdot$ J.-M. Juang $\cdot$ Y.-B. Liu $\cdot$ Y.-L. Ho

W.-J. Chen · S. K. S. Huang · J.-L. Lin $(\square)$

Department of Internal Medicine, National Taiwan University

Hospital, No. 7, Chung-Shan S Road, Taipei, Taiwan

E-mail: jiunn@ha.mc.ntu.edu.tw

Tel: + 886-2-23123456

Fax: + 886-2-23951841

S.-J. Yeh · C.-C. Wang · Y.-L. Ko

Department of Medicine, Chang Gung Memorial Hospital, Taipei, Taiwan
T.-J. Wu

Taichung Veterans General Hospital, School of Medicine,

National Yang-Ming University, Taipei, Taiwan

K.-C. Ueng

Division of Cardiology and Cardiovascular Surgery,

Institute of Medicine, Chung-Shan Medical University Hospital,

Taichung, Taiwan

M.-H. Lei · H.-M. Tsao

Department of Internal Medicine, Poh-Ai Hospital, Lotung, Taiwan

S.-A. Chen

School of Medicine, Taipei Veterans General Hospital,

National Yang-Ming University, Taipei, Taiwan

T.-K. Lin

Cardiovascular Division, Department of Internal Medicine,

Buddhist Dalin Tzu Chi General Hospital, Dalin, Taiwan

M.-H. Wu

Department of Pediatrics, National Taiwan University Hospital,

Taipei, Taiwan

H.-M. Lo

Department of Internal Medicine, Shin Kong Wu Ho-Su Memorial Hospital, Taipei, Taiwan 